\title{
Identification of Formal Fallacies in a Natural Dialogue
}

\author{
Magdalena Kacprzak ${ }^{1}$ and Anna Sawicka ${ }^{2}$ \\ 1 Faculty of Computer Science, Białystok University of Technology, Poland \\ 2 Faculty of Computer Science, Polish-Japanese Institute of Information Technology, \\ Warsaw, Poland
}

\begin{abstract}
This paper is a continuation of the work presented at CS\&P 2012 where the LND dialogue system was proposed. It brings together and unifies two traditions in studying dialogue as a game: the dialogical logic introduced by Lorenzen and persuasion dialogue games as specified by Prakken. The aim of the system LND is to recognize and verify formal fallacies in dialogues. Now we extend this system with a new protocol which allows for reconstruction of natural dialogues in which parties can be committed to formal fallacies. Finally, we show the implementation of the applied protocols.
\end{abstract}

Keywords: protocol for dialogue games, natural dialogue, formal fallacy, Lorenzen Natural Dialogue

\section{Introduction}

In [19], Yaskorska, Budzynska and Kacprzak proposed a protocol, called LND, for verifying during a dialogue whether a propositional formula is a tautology. This protocol is based on Lorenzen's dialogical logic $[7,8,15]$. The aim of this protocol is to use it in a game simulating natural dialogue as an inference scheme validator. Participants of dialogues perform a variety of actions. Some of them can be recognized as justification of player's standpoint. Such an argumentation may refer to argumentation schemes based on propositional tautologies. The LND game tests propositional formulas and thereby decides whether a corresponding inference is correct. The contribution of the present work is to introduce a dialogue system, called PND, in which players are allowed to make formal fallacies, that is, those that use schemes which are not equivalent to a valid formulas of the underlying logic. In our approach, we limit ourselves to propositional calculus and use as a departing point the general framework for dialogues for argumentation proposed by Prakken $[13,14]$. We also define rules which determine how LND games can be nested in PND games. The result is a dialogue system in which players can be committed to formal fallacies, and can identify, verify and debug them.

In many of initially studied dialogue systems like for example the one proposed by Hamblin [6] as well as currently defined systems, arguments can be 
constructed only in accordance with the assumed logical base. As a result, players do not have the possibilities of making a formal error in contrast to the participants of natural, real-life dialogues. This work complements the gap. The idea of combining Lorenzen's games with natural persuasion dialogues was also studied by Walton and Krabbe [17]. They proposed Complex Persuasion Dialogue (CPD) which embeds Lorenzen-like dialogue into Hamblin-like dialogue. However, their motivation was to allow players to help their opponents to infer conclusions logically following from their commitments rather then identify formal fallacies.

The study of the argumentation dialogues is of particular interest in areas such as artificial intelligence and multi-agent systems $[1,11]$. The research program that combines dialogue theory and argumentation theory with the new four-valued approach to modeling multi-agent inter-actions is guided by a Polish group of scientists. Their discussion on an implementation of four speech acts: assert, concede, request and challenge in a paraconsistent framework is presented in [4]. In the paper [3], they show how speech acts and agents' reasoning rules naturally combine in the framework of $4 \mathrm{QL}$ [16], leading to intuitive conclusions while maintaining tractability. Thereby they justify that this four-valued approach can be applied to modelling complex dialogues and argumentations between agents, reasoning in uncertain and dynamic environments. The semantics of speech acts which are applied in deliberation dialogues and thereby are used for modelling communication in teamwork is studied in [5].

The rest of the article is organized as follows. Section 2 presents Prakken's general framework for argumentation dialogue games. In Sect. 3 the extension of this framework, called PND, is introduced. It allows for modelling dialogues in which inference rules used by players are publicly declared and can be challenged. In Sect. 4 the LND system for testing propositional tautologies during a dialogue is described. In Sect. 5 the rules for embedding LND into PND are defined. Finally, in Sect. 6 and 7 the implementation of our protocols and conclusion remarks, respectively, are discussed. The Appendix provides locution, protocol and effect rules for LND games.

\section{Formal Framework for Dialogue Games}

In [13], Prakken proposes a dialogue game which defines principles of argumentation dialogues, i.e., rules governing the meaning and the use of speech acts. In this system, dialogue utterances are treated as moves in a game and rules of their appropriateness are formulated as rules of the game. Prakken's system provides a basis for our new argumentation dialogue system. The reason why we choose this system from other dialog systems $[10,12,17]$ is that it covers a class of argumentation dialogues rather then one selected kind of a dialogue. Moreover this system is flexible with respect to the applied underlying logics, alternative sets of locutions and more or less strict locution rules. Thereby, it offers a nice basis for further research and extensions. 
Below, the main terms and definitions of a formal framework of dialogue games for argumentation, introduced by Prakken in [13], are quoted. In the next section, the modification of this system is shown.

All dialogues of the system are assumed to be for two parties arguing about a single dialogue topic $t$, the proponent $P$ who defeats $t$ and the opponent $O$ who challenges $t$. Both proponent and opponent are equipped with a set of commitments that are understood as publicly incurred standpoints. Commitments are expected to be defended upon a challenge.

Definition 1. A dialogue system for argumentation is a pair $(\mathcal{L}, \mathcal{D})$, where $\mathcal{L}$ is a logic for argumentation and $\mathcal{D}$ is a dialogue system proper.

The elements of the above top level definition are in turn defined as follows.

Definition 2. A logic for argumentation $\mathcal{L}$ is a tuple $\left(L_{t}, R\right.$, Args,$\left.\rightarrow\right)$, where $L_{t}$ is a logical language called the topic language, $R$ is a set of inference rules over $L_{t}$, Args is a set of arguments, and $\rightarrow$ is a binary relation of defeat defined on Args.

For any argument $A \in A r g s, \operatorname{prem}(A)$ is the set of premises of $A$ and $\operatorname{conc}(A)$ is the conclusion of $A$.

Definition 3. An argumentation theory $T_{F}$ within $\mathcal{L}$ (where $F \subset L_{t}$ ) is a pair $\left(A, \rightarrow_{/ A}\right)$ where $A$ consists of all arguments in Args with only premises and conclusions from $F$ and $\rightarrow_{/ A}$ is $\rightarrow$ restricted to $A \times A$. $T_{F}$ is called finitary if none of its arguments has an infinite number of defeaters.

The idea of an argumentation theory is that it contains all arguments that are constructible on the basis of a certain theory.

Definition 4. A dialogue system proper is a triple $\mathcal{D}=\left(L_{c}, \operatorname{Pr}, C\right)$ where $L_{c}$ is a communication language, $\operatorname{Pr}$ is a protocol for $L_{c}$, and $C$ is a set of effect rules of locutions in $L_{c}$.

Below the elements of a dialogue system proper are specified.

Definition 5. A communicating language $L_{c}$ is a set of locutions.

The most frequently considered locutions are: $\operatorname{claim}(\alpha)$ - the speaker asserts that $\alpha$ is the case, why $(\alpha)$ - the speaker challenges $\alpha$ and asks for reasons why it would be the case, concede $(\alpha)$ - the speaker admits that $\alpha$ is the case, $\operatorname{retract}(\alpha)$ - the speaker declares that he is not committed (any more) to $\alpha, \operatorname{argue}(A)-$ the speaker provides an argument $A$, where $\alpha \in L_{t}$ and $A \in$ Args.

The protocol for $L_{c}$ is defined in terms of the notion of a dialogue, which in turn is defined with the notion of a move. The set $M$ of moves is defined as $\mathbb{N} \times\{P, O\} \times L_{c} \times \mathbb{N}$, where the four elements of a move $m$ are respectively denoted by: $i d(m)$ - the identifier of the move, $p l(m)$ - the player of the move, $s(m)$ - the speech act (locution) performed in $m, t(m)$ - the target of $m$. 
The set of dialogues, denoted by $M \leq \infty$, is the set of all sequences $m_{1}, \ldots, m_{i}, \ldots$ from $M$ such that each $i^{t h}$ element in the sequence has identifier $i, t\left(m_{1}\right)=0$, for all $i>1$ it holds that $t\left(m_{i}\right)=j$ for some $m_{j}$ preceding $m_{i}$ in the sequence. The set of finite dialogues, denoted by $M^{<\infty}$, is the set of all finite sequences that satisfy these conditions. When $d$ is a dialogue and $m$ a move, then $(d, m)$ will denote the continuation of $d$ with $m$.

A protocol also assumes a turntaking rule. A turntaking function $T$ is a function $T: M^{<\infty} \rightarrow 2^{\{P, O\}}$ such that $T(\emptyset)=\{P\}$. A turn of a dialogue is a maximal sequence of stages in the dialogue where the same player moves. This definition allows that more than one speaker has the right to speak next.

The key notion for the dialogue system is the protocol.

Definition 6. A protocol on the set of moves $M$ is a set $\operatorname{Pr} \subseteq M^{<\infty}$ satisfying the condition that whenever $d$ is in $\mathrm{Pr}$, so are all initial sequences that $d$ starts with.

A partial function $\overline{\operatorname{Pr}}: M^{<\infty} \rightarrow 2^{M}$ is derived from $\operatorname{Pr}$ as follows: $\overline{\operatorname{Pr}}(d)=$ undefined whenever $d \notin \operatorname{Pr} ; \overline{\operatorname{Pr}}(d)=\{m:(d, m) \in \operatorname{Pr}\}$ otherwise. The elements of the domain $\operatorname{dom}(\overline{\mathrm{Pr}})$ are called the legal finite dialogues. The elements of $\overline{\operatorname{Pr}}(d)$ are called the moves allowed after $d$. If $d$ is a legal dialogue and $\overline{\operatorname{Pr}}(d)=\emptyset$, then $d$ is said to be a terminated dialogue.

All protocols of Prakken's system are assumed to satisfy the following conditions for all moves $m$ and all legal finite dialogues $d$. If $m \in \overline{\operatorname{Pr}}(d)$, then:

PP1 $p l(m) \in T(d)$,

PP2 If $d \neq d_{0}$ and $m \neq m_{1}$, then $s(m)$ is a reply to $s(t(m))$ according to $L_{c}$,

PP3 If $m$ replies to $m^{\prime}$, then $p l(m) \neq p l\left(m^{\prime}\right)$,

PP4 If there is an $m^{\prime}$ in $d$ such that $t(m)=t\left(m^{\prime}\right)$, then $s(m) \neq s\left(m^{\prime}\right)$,

PP5 For any $m^{\prime} \in d$ that surrenders to $t(m), m^{\prime}$ is not an attacking counterpart of $m$.

Rule PP1 says that a move is legal only if moved by the player-to-move. PP2 says that a replying move must be a reply to its target according to $L_{c}$. PP3 says that one cannot reply to one's own moves. Rule PP4 states that if the player backtracks, the new move must be different from the first one. Finally, PP5 says that surrenders should not be 'revoked'.

Every utterance from $L_{c}$ can influence participants' commitments. Results of utterances are determined by commitment rules which are specified as a commitment function.

Definition 7. A commitment function is a function: $C: M^{<\infty} \times\{P, O\} \rightarrow 2^{L_{t}}$, such that $C(\emptyset, i)=\emptyset$ for $i \in\{P, O\}$.

$C(d, i)$, for a participant $i \in\{P, O\}$ and a stage of a dialogue $d \in M^{<\infty}$, denotes a player $i$ 's commitments at the stage of a dialogue $d$. 


\section{Prakken Natural Dialogue}

The framework for dialogue games proposed by Prakken implements the intention of all argumentation dialogue games, that is, to define rules that allow participants to play the dialogue in a way that could lead to an agreement. In a persuasion dialogue game it is understood as an opportunity to convince the opponent to change its position, and consequently resolve a conflict of opinion. Therefore, much attention has been devoted to establishing conditions under which such an agreement can be achieved. In Prakken's system it is assumed that all participants agree to the topic language and the set of rules under which valid arguments are defined. What is more, all the rules are correct in the assumed logic. Unquestionably, this is the basis for the agreement. Observe, however, that participants of real life dialogues very rarely determine among themselves their knowledge base or rules they use. Moreover, they are often committed to wrong inferences. The case when the participants apply different, not necessarily correct, inference schemes cannot be modelled in Prakken's system. This is why we propose some modifications of his system. In Prakken's general framework, players can argue using one of the possible arguments. All the arguments are constructed over inference rules of the assumed logical system. Thereby they are correct. Our proposition is to allow players to perform locutions in which incorrect argumentation is provided.

The new dialogue system, is called Prakken Natural Dialogue (PND) and is a pair $(\mathcal{L}, \mathcal{D})$. A logic for argumentation $\mathcal{L}$ is a tuple $\left(L_{t}, R, \operatorname{Args}, \rightarrow\right)$ where $L_{t}$ is a propositional logic and $R$ is as set of inference rules of $L_{t}$. To realize our goal, we need to distinguish in the topic language two sentences: (a) "The formula $\theta$ is a propositional tautology" and (b) "The formula $\theta$ is obtained from the formula $\psi$ by some substitution". For convenience, we introduce the following abbreviations. Let $\operatorname{Taut}(\theta)$ will be short for " $\theta$ is a propositional tautology". This sentence should be true or false. We do not state here that actually $\theta$ is a tautology. Moreover, if $\theta\left(q_{1}, \ldots, q_{n}\right)$ is a propositional formula build under propositions $q_{1}, \ldots, q_{n}$ and $\alpha_{1}, \ldots, \alpha_{n}$ are propositional formulas, we write $\theta\left(\alpha_{1} /\right.$ $\left.q_{1}, \ldots, \alpha_{n} / q_{n}\right)$ for a formula $\theta$ in which the proposition $q_{i}$ is replaced with the formula $\alpha_{i}$ for $i=1, \ldots, n$. It is obvious that if $\operatorname{Taut}\left(\theta\left(q_{1}, \ldots, q_{n}\right)\right)$ is true (i.e. $\theta\left(q_{1}, \ldots, q_{n}\right)$ is a tautology), then $\operatorname{Taut}\left(\theta\left(\alpha_{1} / q_{1}, \ldots, \alpha_{n} / q_{n}\right)\right)$ is also true (i.e. $\theta\left(\alpha_{1} / q_{1}, \ldots, \alpha_{n} / q_{n}\right)$ is a tautology too $)$. We will also write $\theta\left(\alpha_{1}\left(p_{1}, \ldots, p_{k}\right) /\right.$ $\left.q_{1}, \ldots, \alpha_{n}\left(p_{1}, \ldots, p_{k}\right) / q_{n}\right)=\psi\left(p_{1}, \ldots, p_{k}\right)$ if the formula $\psi$ is obtained from $\theta$ by substitution $q_{i}$ for $\alpha_{i}(i=1, \ldots, n)$.

The set of arguments Args is a set of pairs $A=(\operatorname{Prem}(A), \operatorname{Conc}(A))$ where $\operatorname{prem}(A)$ is a set of premises (a finite set of propositional formulas) and $\operatorname{conc}(A)$ is a conclusion (a propositional formula) such that the formula $\bigwedge_{a \in \operatorname{prem}(A)} \Rightarrow$ $\operatorname{conc}(A)$ is a propositional tautology. Since in this paper we do not focus on attacks and counterattacks on arguments, we omit here the specification of the defeat relation.

A dialogue system proper for $\mathrm{PND}, \mathcal{D}=\left(L_{c}, P, C_{P}, C_{O}\right)$ is defined by locution, protocol, and effect rules presented in the next subsections. Taking into account the structural properties [9], the protocol for PND is: 
- unique-move, i.e., the turn switches after each move,

- multi-reply, i.e., players can return to earlier choices and try alternative moves to the other player's moves,

- immediate-reply, i.e., each player must immediately respond to the move of the other player.

\subsection{Locution rules}

The communication language of PND assumes the following locutions:

PL1 Claim $\operatorname{claim}(\varphi)$ is performed when a player asserts that sentence $\varphi$ is true and his antagonist does not have this sentence in his commitment base.

PL2 Concession concede $(\varphi)$ is performed when a player asserts that sentence $\varphi$ is true and his antagonist has this sentence in his commitment base.

PL3 Challenge $w h y(\varphi)$ is performed when a player asks about a proof for $\varphi$.

PL4 Argumentation $(\varphi)$ since $\left(\psi_{1}, \ldots, \psi_{n}\right.$, Taut $\left.(\theta)\right)$ is performed when a player justifies statement $\varphi$ with a set of premises $\psi_{1}, \ldots, \psi_{n}$ and the inference rule corresponding to the formula $\theta$. A player can use in this locution a rule which is not correct and does not correspond to a tautology.

PL5 Retraction $\operatorname{retract}(\varphi)$ is performed when a player resigns from the statement that sentence $\varphi$ is true.

\section{$3.2 \quad$ Protocol rules}

The protocol for PND satisfies the protocol rules PP1-PP5 of Prakken's general framework and adds the following, where $s \in\{P, O\}, d \in \operatorname{Pr}, m \in \overline{\operatorname{Pr}}(d)$, $\varphi, \psi_{1}, \ldots, \psi_{n}, \Theta \in L_{t}$ :

PP6 if $d=\emptyset$, then $s(m)$ is of the form

(a) $\operatorname{claim}(\varphi)$ or

(b) $(\varphi)$ since $\left(\psi_{1}, \ldots, \psi_{n}\right.$, Taut $\left.(\theta)\right)$,

PP7 if $m$ concedes the conclusion of an argument moved in $m^{\prime}$, then $m^{\prime}$ does not reply to a why move,

PP8 if $s(m)$ is $\operatorname{claim}(\varphi)$, then $s\left(m^{\prime}\right)$ for $m^{\prime} \in \overline{\operatorname{Pr}}((d, m))$ is of the form

(a) $w h y(\varphi)$ (attack) or

(b) concede $(\varphi)$ (surrender),

PP9 if $s(m)$ is why $(\varphi)$, then $s\left(m^{\prime}\right)$ for $m^{\prime} \in \overline{\operatorname{Pr}}((d, m))$ is of the form

(a) $(\varphi)$ since $\left(\psi_{1}, \ldots, \psi_{n}, \operatorname{Taut}\left(\psi_{1} \wedge \ldots \wedge \psi_{n} \Rightarrow \varphi\right)\right)$ (attack) or

(b) $(\varphi)$ since $\left(\operatorname{Taut}\left(\theta\left(q_{1}, \ldots, q_{n}\right)\right), \operatorname{Taut}\left(\theta\left(\alpha_{1} / q_{1}, \ldots, \alpha_{n} / q_{n}\right)\right)=\beta\right)$ for some formulas $\alpha_{1}, \ldots, \alpha_{n}$ if $\varphi=\operatorname{Taut}(\beta)$ (attack) or

(c) $\operatorname{retract}(\varphi)$ (surrender),

PP10 if $s(m)$ is $(\varphi)$ since $\left(\psi_{1}, \ldots, \psi_{n}, \operatorname{Taut}(\theta)\right)$, then $s\left(m^{\prime}\right)$ for $m^{\prime} \in \overline{\operatorname{Pr}}((d, m))$ is of the form

(a) why $(\alpha)$ where $\alpha \in\left\{\psi_{1}, \ldots, \psi_{n}, \operatorname{Taut}(\theta)\right\}$ (attack) or

(b) $(\neg \varphi)$ since $\left(\beta_{1}, \ldots, \beta_{n}\right.$, Taut $\left.\left(\beta_{1} \wedge \ldots \wedge \beta_{n} \Rightarrow \neg \varphi\right)\right)$

(c) concede $(\alpha)$ where $\alpha \in\left\{\varphi, \psi_{1}, \ldots, \psi_{n}, \operatorname{Taut}(\theta)\right\}$ (surrender), 
PP11 if $s(m)$ is concede $(\varphi)$ or $\operatorname{retract}(\varphi)$, then $s\left(m^{\prime}\right)$ for $m^{\prime} \in \overline{\operatorname{Pr}}((d, m))$ is

(a) a reply (attack or surrender) to some earlier move of the other player or

(b) $\overline{\operatorname{Pr}}((d, m))=\emptyset$.

Rules PP6 and PP7 are inspired by liberal dialogues (see [13]). PP6 says that each dialogue begins with either a claim or an argument. The initial claim or, if a dialogue starts with an argument, its conclusion is the topic of the dialogue. PP7 restricts concessions of an argument conclusion to conclusions of counterarguments. Rules PP8-PP11 describe possible moves after specific locution. Observe that every move replies to some earlier move of the antagonist and it is either attack or surrender.

\subsection{Effect rules}

In PND there are two participants. Therefore, we need to define a commitment functions for both of them:

$$
C_{s}: M^{<\infty} \times\{P, O\} \rightarrow 2^{L_{t}}
$$

where $s \in\{P, O\}$. However, locution rules do not depend on the role which the performer of the locution plays. Effects on the commitment sets after execution specific moves are described below, where $s$ denotes the speaker, $\left(m_{0}, \ldots, m_{n}\right)$ is a legal dialogue, and $\left.\varphi, \psi_{1}, \ldots, \psi_{n}, \operatorname{Taut}(\Theta)\right) \in L_{t}$.

PE1 if $s\left(m_{n}\right)=\operatorname{claim}(\varphi)$, then $C_{s}\left(m_{0}, m_{1}, \ldots, m_{n}\right)=C_{s}\left(m_{0}, m_{1}, \ldots, m_{n-1}\right) \cup$ $\{\varphi\}$, i.e. after $\operatorname{claim}(\varphi)$ the formula $\varphi$ is added to the $s$ 's commitment set,

PE2 if $s\left(m_{n}\right)=$ concede $(\varphi)$, then $C_{s}\left(m_{0}, \ldots, m_{n}\right)=C_{s}\left(m_{0}, \ldots, m_{n-1}\right) \cup\{\varphi\}$, i.e., after concede $(\varphi)$ the formula $\varphi$ is added to the $s$ 's commitment set,

PE3 if $s\left(m_{n}\right)=w h y(\varphi)$, then $C_{s}\left(m_{0}, m_{1}, \ldots, m_{n}\right)=C_{s}\left(m_{0}, m_{1}, \ldots, m_{n-1}\right)$, i.e., after $w h y(\varphi)$ the $s$ 's commitment set does not change,

PE4 if $s\left(m_{n}\right)=(\varphi)$ since $\left(\psi_{1}, \ldots, \psi_{n}, \operatorname{Taut}(\theta)\right)$, then $C_{s}\left(m_{0}, m_{1}, \ldots, m_{n}\right)=$ $C_{s}\left(m_{0}, m_{1}, \ldots, m_{n-1}\right) \cup\left\{\varphi, \psi_{1}, \ldots, \psi_{n}, \operatorname{Taut}(\theta)\right\}$, i.e., after this locution the formulas $\varphi, \psi_{1}, \ldots, \psi_{n}, \operatorname{Taut}(\theta)$ are added to $s$ 's commitment set,

PE5 if $s\left(m_{n}\right)=\operatorname{retract}(\varphi)$, then $C_{s}\left(m_{0}, m_{1}, \ldots, m_{n}\right)=C_{s}\left(m_{0}, m_{1}, \ldots, m_{n-1}\right) \backslash\{\varphi\}$, i.e., after retract $(\varphi)$ the formula $\varphi$ is deleted from the $s$ 's commitment set.

\subsection{Turntaking}

In a PND game, $P$ makes the first move, then $O$ and $P$ take turns in performing moves. Thus the turntaking function is defined as follows:

$$
T\left(m_{0}, m_{1}, \ldots, m_{n}\right)=\left\{\begin{array}{l}
P \text { iff } n \text { is even } \\
O \text { iff } n \text { is odd }
\end{array} .\right.
$$




\section{Lorenzen Natural Dialogue}

In [18], Yaskorska, Budzynska, and Kacprzak proposed a dialogue system, LND, that allows communicating agents to prove that a formula used in an argument is a classical propositional tautology, and, as a result, to identify and eliminate classical propositional formal fallacies committed during a natural dialogue. This is achieved through a combination of a system for representing natural dialogues with a system for representing formal dialogues. In the first case, the framework proposed by Prakken [14] was used, since it provides a generic and formal specification of the main elements of dialogue systems for persuasion. For handling formal fallacies in a dialogue, the dialogical logic introduced by Lorenzen $[7,8$, $15]$ was applied. Lorenzen's dialogue games allow the players to prove that a formula is a tautology of classical propositional logic, if the proponent has a winning strategy in a given game. The aim of this system is not to jointly built an argument: $\varphi$, therefore $\psi$, as in inquiry dialogues (see e.g. [2]), but to allow the participants to play against each other starting with opposing viewpoints on an argument validity and determining which player wins.

The dialogical logic communication language and structure are different from systems for natural dialogues. For example, in Lorenzen's system the only moves available to speakers are: $X$ attacks $\varphi$ and $X$ defends $\varphi$, while, according to Prakken's specification, in systems for natural dialogues the legal locutions can be: claim $\varphi$, why $\varphi$, concede $\varphi$, retract $\varphi, \varphi$ since $S$, question $\varphi$. Therefore the main challenge was to introduce a new description of the dialogical logic which meets the requirements of Prakken's generic specification. The correspondence result between the original and the new version of the dialogical logic is presented in [19] where it is proved that a winning strategy for a proponent in the original version of the dialogical logic means a winning strategy for a proponent in the new one, and conversely.

The locution, protocol and effect rules of the LND system are presented in the Appendix.

\section{$5 \quad$ Embedding Dialogues}

The aim of dialogical logic introduced by Lorenzen is to define logical connectives in terms of attacks and defences, and then determined whether the formula under discussion is valid in the given logical system. The goal of argumentation dialogue systems is to define rules of the game in which participants can challenge and provide reasons for their claims and positions. Our intention is to combine these two approaches. This object is achieved by proposing the system LND and the system PND and by defining rules for their embedding. The main advantage of combining the systems LND and PND is to obtain a uniform system that allows modelling of dialogues in which participants can be committed to formal fallacies and may discuss the patterns of inferences, in terms of their correctness, according to a given logical framework. In this work it is propositional logic.

In the new dialogue system which is a combination of LND with PND, we take the following assumptions: 
- in the PND (LND) part of the game, players use the same topic and communication languages,

- players have different commitment sets in PND game and hypothetical commitment sets in LND game,

- players can use correct and incorrect inference rules and correct and incorrect arguments constructed under these rules,

- players can challenge claims and inference rules of the other player,

- a correct rule is a rule which corresponds to some propositional tautology,

- correctness of inference rules is examining during Lorenzen's game, i.e., if a player challenges some rule, its antagonist starts Lorenzen's game and takes the role of the proponent,

- if the proponent of Lorenzen's game looses, then he must retract from the commitment which says that the inference rule under discussion is correct.

Below the rules for embedding LND into PND are given. Two new locutions are introduced: InitLor and EndLor.

PL6 Initialization The locution InitLor $(\theta)$ breaks the natural dialogue and initializes the DL-like dialogue for formula $\theta$. The player who performed InitLor $(\theta)$ becomes the proponent for $\theta$ in the embedded DL-like dialogue.

PL7 Ending The locution EndLor $(\theta)$ ends the DL-like dialogue for $\theta$ and resumes the broken natural dialogue.

In the approach it is assumed that DL-like dialogue for a formula $\theta$ starts when one of the players challenges this formula. Then, the players examine $\theta$ in accordance with the rules of DL-like games. Protocol rules for embedding a formal dialogue into a natural one is described in PP12 - PP15.

PP12 The locution $\operatorname{InitLor}(\theta)$ can be performed as a reply to the locution why $(\operatorname{Taut}(\theta))$ or the locution $\operatorname{claim}(\neg \operatorname{Taut}(\theta))$ executed in a PND game.

PP13 After the locution InitLor $(\theta)$ players can perform the same actions which are allowed to execute after $\operatorname{claim}(\theta)$ according to the protocol rules P1-P8 of the system LND (see Appendix).

PP14 The locution EndLor $(\theta)$ can be performed by a player $X$ if $X$ has no legal move according to the protocol rules P1-P8 of the system LND (see Appendix).

PP15 After the locution EndLor $(\theta),(1)$ if $P$ is the performer, then $P$ executes $\operatorname{retract}(\operatorname{Taut}(\theta))$ in the broken PND game, (2) if $O$ is the performer, then $O$ executes concede $(\operatorname{Taut}(\theta))$ in the broken PND game.

\section{Implementation}

The implementation consists of two applications: LNDGame and PNDGame. The program LNDGame is intended to implement the dialogue construction based on the LND game. All the basic notions are modelled in the program in a direct way. According to the adopted formal definitions, a dialogue $D(\theta)$, for 
a formula $\theta$, is a set of dialogue games consisting of sequences of moves. The initial move is performed by the proponent, which claims formula $\theta$. During the dialogue game, each participant makes moves, one after the other, according to the rules of the protocol.

In the program, a move is defined by locution type and the formula, it also has a reference to the locution it responds to. A formula is represented as a tree of subformulas, even though for the user it appears rather as a sequence of symbols. Initial formula proposed by the proponent can be given in two ways: by providing a sequence of symbols or by recursively constructing subformulas using GUI. A hypothetical commitment set is associated with each participant. It is an increasing set of formulas previously committed.

The application enables two modes. In the first, interactive mode, the user has a possibility to choose each move on the behalf of one of the participants. The move can be chosen from the list of possible moves updated after each move. The result is a sequence of moves chosen by a participant at each step of one of the dialogue games, and the result of the game, i.e., whether the proponent wins the game or not. In the second, auto mode, program can scan all possible dialogue games for the specified initial formula $\theta$. The result contains a sequences of moves, one for each theoretically possible dialogue game, with information about the other available moves at each step. If a proponent wins all the possible dialogue games, it wins the dialogue $D(\theta)$ (in this case formula $\theta$ is a tautology). Certainly, we have to take into account the complexity of such a scan for more complex formulas. The current version is adapted to handle real-life size formulas and illustrates that even for small formulas used in short dialogues $D(\theta)$ can be very large.

The second application, PNDGame, is intended to implement the dialogue games based on PND. During the dialogue game, understood in the same way as above, each of the participants can use different inference schemes, some of them can be even incorrect. Each participant can also challenge another participant's rule of inference using Lorenzen-style Natural Dialogue (and the same mechanism as proposed in LNDGame application). To prove that the rule is incorrect, challenging participant has to start a Lorenzen game and to win it. Then, the proponent of the rule has to withdraw this rule from his set of inference rules.

The implementation was made in Java language, which will facilitate further development of application, and also software portability. This choice helps us to avoid from the restrictions of other protocols than analyzed in this paper. The participants of the dialog, as well as game manager, are implemented as a separated, eventually distributed over the network classes. The aim of this implementation is dialogue game simulation and a decision making support during such a game. It allows to verify the validity of formulas used in dialogues as tautologies and to identify formal fallacies. It also enables recording of conducted dialogues games, which makes later analysis possible. Subsequent versions of the application prepared by the authors will correspond to the efforts to combine several formal systems for modelling natural dialogues in terms of games and analyzing properties of such dialogue games. 


\section{Conclusions}

This work provides a unified dialogue system for argumentation which combines two approaches: Lorenzen's dialogical logic (DL) with a modified Prakken's framework for dialogue games for argumentation. The idea of dialogical logic was applied in Lorenzen Natural Dialogue (LND) where the structural and particle rules of DL were reconstructed and defined in terms of locution, commitment and protocol rules of dialogue games [19]. Prakken's system was extended with specific locutions which allows players to use incorrect arguments, to show directly the inferences on which these arguments are based and to challenge them. The result is a Prakken Natural Dialogue. Finally, the rules for embedding LND into PND are defined.

The main advantage of the new system is that in the course of a dialogue the participants can verify their sets of rules and create new arguments. Thereby, this idea allows a study argumentation systems in which participants have the ability to learn. The dynamic nature of dialogues and frequent change of information may be reflected not only in revising beliefs and commitments of players but also in changing the way in which they argue and reason.

The proposed system can be used both as a simulation of natural dialogues conducted in artificial intelligence systems, and as a tool for argumentation and persuasion communication in multi-agent systems.

\section{References}

1. Amgoud, L., Maudet, N., Parsons, S.: Modelling dialogues using argumentation. In: Proc. of the Fourth Int. Conf. on Mulit-Agent Systems (2000) 31-38

2. Black, E., Hunter, A.: An inquiry dialogue system. Autonomous Agent Multi-Agent Systems 18 (2009) 173-209

3. Dunin-Kȩplicz, B., Strachocka, A., Szałas, A., Verbrugge, R.: A paraconsistent approach to speech acts. In: Proc. of ArgMAS (2012) 59-78

4. Dunin-Kȩplicz, B., Strachocka, A., Szałas, A., Verbrugge, R.: Perceiving speech acts under incomplete and inconsistent information. Frontiers in Artificial Intelligence and Applications 252 (2013) 255 - 264

5. Dunin-Kęplicz, B., Strachocka, A., Verbrugge, R.: Deliberation dialogues during multiagent planning. LNCS $\mathbf{6 8 0 4}$ (2011) 170-181

6. Hamblin, C.: Fallacies. Methuen, London (1970)

7. Keiff, L.: Dialogical logic. The Stanford Encyclopedia of Philosophy (2011)

8. Lorenz, K., Lorenzen, P.: Dialogische logik. WBG. Darmstadt (1978)

9. Loui, R.: Process and policy: resource-bounded non-demonstrative reasoning. Computational Intelligence 14 (1998) 1-38

10. Mackenzie, J.D.: Question begging in non-cumulative systems. Journal of Philosophical Logic 8 (1979) 117-133

11. McBurney, P., Parsons, S.: Games that agents play: A formal framework for dialogues between autonomous agents. Journal of Logic, Language and Information $\mathbf{1 3}$ (2002) 315-343

12. Parsons, S., Wooldridge, M., Amgoud, L.: Properties ans complexity of some formal inter-agent dialogues. Journal of Logic and Computation 13 (2003) 347-376 
13. Prakken, H.: Coherence and flexibility in dialogue games for argumentation. Journal of Logic and Computation 15 (2005) 1009-1040

14. Prakken, H.: Formal systems for persuasion dialogue. The Knowledge Engineering Review 21 (2006) 163-188

15. Rahman, S., Tulenheimo, T.: From games to dialogues and back: towards a general frame for validity. Games: Unifying Logic, Language, and Philosophy (2006)

16. Szałas, A.: How an agent might think. Logic J. IGPL 21(3) (2013) 515-535

17. Walton, D.N., Krabbe, E.C.W.: Commitment in Dialogue: Basic Concepts of Interpersonal Reasoning. State University of N.Y. Press (1995)

18. Yaskorska, O., Budzynska, K., Kacprzak, M.: Rules for formal and natural dialogues in agent communication. In: Proc. of CS\&P (2012) 416-427

19. Yaskorska, O., Budzynska, K., Kacprzak, M.: Proving propositional tautologies in a natural dialogue. Fundamenta Informaticae (2013)

\section{Appendix}

In LND game the set of players consists of two elements $\{\mathbf{O}, \mathbf{P}\}$. Topic language $L_{t}$ is assumed to be that of classical propositional logic. The dialogue system proper is specified by the locution, protocol and effect rules.

Locution rules. The locution rules for LND are specified as follows: [L1] Claim claim $\varphi$ is performed when a player: (1) attacks $\neg A$, then $\varphi$ is a formula $A,(2)$ defends $A \wedge B$, then $\varphi$ is a formula $A$ or a formula $B,(3)$ attacks $A \rightarrow B$, then $\varphi$ is a formula $A$, (4) defends $A \rightarrow B$, then $\varphi$ is a formula $B$; [L2] Concession concede $\varphi$ can be performed only by a proponent $\mathbf{P}$, and this locution is performed when $\varphi$ is an atomic formula and the performer: (1) attacks $\neg A$, then $\varphi$ is a formula $A$, (2) defends $A \wedge B$, then $\varphi$ is a formula $A$ or a formula $B,(3)$ attacks $A \rightarrow B$, then $\varphi$ is a formula $A$, (4) defends $A \rightarrow B$, then $\varphi$ is a formula $B$; [L3] Argumentation $\varphi$ since $\psi$ is performed when a player defends $A \vee B$, then $\varphi$ is a formula $A \vee B$ and $\psi$ is a set which includes the formula $A$ or the formula $B$; $[\mathbf{L} 4]$ Challenge The challenge why $\varphi$ is performed when a player attacks $A \vee B$, then $\varphi$ is a formula $A \vee B$; [L5] Question The question question $\varphi$ is performed when a player attacks $A \wedge B$, then $\varphi$ is a formula $A$ or a formula $B$.

Protocol rules. The LND protocol descries a formal dialogue game $\triangle=m_{0}, \ldots, m_{n}$ on a topic $A$, which is called a DL-like game. Let $\mathbf{D}^{\prime}(A)$ be DL-like dialogue for $A$, i.e. a set of DL-like games for $A$. The protocol is specified as follows: [P1] In the first move $\mathbf{P}$ performs claim $\varphi$ where $\varphi$ is the topic $A$; next players perform one locution at each turn; [P2] A player $\mathbf{P}$ cannot perform $\operatorname{claim} \varphi$ where $\varphi$ is a proposition; he can state that $\varphi$ is true executing concede $\varphi$ but this move can be performed only if $\mathbf{O}$ claimed $\varphi$ in some previous move; [P3] After $\operatorname{claim} \varphi$ a player can perform: (1)cclaim $\psi$, if (a) $\varphi$ is a negation of the formula and $\psi$ is a contradiction to $\varphi,(\mathrm{b}) \varphi$ is the implication and $\psi$ is the antecedent of $\varphi$, (c) $\varphi$ is the antecedent of an implication under the attack and $\psi$ is the consequent of this implication (in P3.1, $\mathrm{P}$ has to follow the restriction described in P2), (2) concede $\psi$, if $\mathbf{P}$ is the player and $\psi$ is a proposition, and (a) $\varphi$ is a negation of the formula and $\psi$ is a contradiction to $\varphi$, (b) if $\varphi$ is the implication under the attack and $\psi$ is its consequent, (3) question $\psi$, if $\varphi$ is a conjunction and $\psi$ is one of its operands, (4) why $\varphi$, if $\varphi$ is a disjunction, (5) attack or defence of any formula uttered before, if $\mathbf{P}$ is the player, (6) no move, if (a) claim $\varphi$ is an attack on negation and $\varphi$ is a proposition, (b) claim $\varphi$ is a defence executed by $\mathbf{P}$, and $\mathbf{O}$ has attacked this defence before; [P4] After concede $\varphi$ performed by $\mathbf{P}$, where $\varphi$ is a proposition, $\mathbf{O}$ has no move; [P5] After $\varphi$ since $\Psi$, where $\Psi=\{\psi\}$ the player can 
perform: (1) $\operatorname{claim} \varphi$, if (a) $\psi$ is a negation of the formula and $\varphi$ is a contradiction to $\psi$, (b) if $\psi$ is the implication $\varphi$ is its antecedent (in P5.1, $\mathrm{P}$ has to follow the restriction described in P2), (2) concede $\varphi$, if $\mathbf{P}$ is the player and $\varphi$ is a proposition, and (a) $\psi$ is a negation of the formula and $\varphi$ is a contradiction to $\psi$, (b) if $\psi$ is the implication under the attack and $\varphi$ is its consequent, (3) question $\varphi$, if $\psi$ is a conjunction and $\varphi$ is one of its operands, (4) why $\psi$, if $\psi$ is a disjunction, (5) attack or defence of any formula uttered before, if $\mathbf{P}$ is the player, (6) no move, if $\varphi$ since $\Psi$ is a defence executed by $\mathbf{P}$, and $\mathbf{O}$ has attacked this defence before; [P6] After why $\varphi$ a player can perform: (1)c $\varphi$ since $\psi$ ( $\mathbf{P}$ has to follow the restriction described in $\mathbf{P 2}),(2)$ attack or defence of any formula uttered before, if $\mathbf{P}$ is the player; [P7] After question $\varphi$ a player can perform: (1)cclaim $\varphi$ ( $\mathbf{P}$ has to follow the restriction described in $\mathbf{P 2}),(2)$ concede $\varphi$, if $\mathbf{P}$ is the player and $\varphi$ is a proposition, (3) attack or defence of any formula uttered before, if $\mathbf{P}$ is the player; [P8] If $\mathbf{O}$ loses a game $\triangle$ which involves the propositional choice made by $\mathbf{O}$ (see DL-rule $\mathbf{S R - 2}$ ), then $\mathbf{O}$ can start a sub-game $\triangle /$. There are three types of sub-games $\triangle$ possible: (I) Assume that $\mathbf{P}$ executes claim $\varphi$ in $\triangle$, where $\varphi$ is $\psi \wedge \psi$, and $\mathbf{O}$ attacks the conjunction by stating: question $\psi$ (the propositional choice step). If they continue to play the game $\triangle$ according to the LND rules and $\mathbf{P}$ makes the last available move, then $\mathbf{O}$ can extend $\triangle$ with a sub-game $\triangle$ by attacking the conjunction one more time using the locution: question $\psi$. (II) Assume that $\mathbf{O}$ executes claim $\varphi$ in $\triangle$, where $\varphi$ is $\psi \vee \psi \prime$. In the next moves, $\mathbf{P}$ attacks the disjunction by stating: why $\varphi$, and $\mathbf{O}$ defends it by stating: $\varphi$ since $\psi$ (the propositional choice step). If they continue to play the game $\triangle$ according to the LND rules and $\mathbf{P}$ makes the last available move, then $\mathbf{O}$ can extend $\triangle$ with a sub-game $\triangle$ ' by defending the disjunction one more time with the locution: $\varphi$ since $\psi$. (III) Assume that in a game $\triangle$, $\mathbf{O}$ executes claim $\varphi$, where $\varphi$ is $\psi \rightarrow \psi \prime$, and $\mathbf{P}$ attacks the implication by stating: claim $\psi$. There are two possible sub-cases: (1) Let $\mathbf{O}$ respond to this attack by defending the implication, i.e., he performs: claim $\psi^{\prime}$ (the propositional choice step). If they continue to play the game $\triangle$ according to the LND rules and $\mathbf{P}$ makes the last available move, then $\mathbf{O}$ can extend $\triangle$ with a sub-game $\triangle$ ' by responding to $\mathbf{P}$ 's attack one more time and attacking the propositional content of $\mathbf{P}$ 's attack, $\psi$, accordingly to its logical form. (2) Let $\mathbf{O}$ respond to $\mathbf{P}$ 's attack by attacking its content, $\psi$, accordingly to its logical form (the propositional choice step). If they continue to play the game $\triangle$ according to the LND rules and $\mathbf{P}$ makes the last available move, $\mathbf{O}$ can extend $\triangle$ with a sub-game $\triangle \prime$ by responding to $\mathbf{P}$ 's attack one more time and defend the implication using the locution: claim $\psi$ '. In all cases P8.I-P8.III, during $\triangle /$ the players may use all the LND rules with a limitation on the $\mathbf{P 2}$ rule such that $\mathbf{P}$ cannot perform concede $\phi$ if $\mathbf{O}$ did not introduce a proposition $\phi$ in $\triangle$ before the propositional choice step and did not introduce a proposition $\phi$ in $\triangle$ '

Effect rules The dynamics of participants' commitments in LND formal games is showed by a hypothetical commitment base. During the game, new formulas are added to this base and no formulas are deleted. For a formal game $\triangle=m_{0}, \ldots, m_{n} \in$ $\mathbf{D}^{\prime}(A)$, the rules for hypothetical commitment base $C_{s}^{\prime}$ of a player $s \in\{\mathbf{O}, \mathbf{P}\}$ are specified below, where $s(m)$ denotes a move of a player $s$ and $\varphi, \psi \in L_{t}$ are propositional formulas: [E1] if $s\left(m_{n}\right)=\operatorname{claim}(\varphi)$, then $C_{s}^{\prime}\left(m_{0}, \ldots, m_{n}\right)=C_{s}^{\prime}\left(m_{0}, \ldots, m_{n-1}\right) \cup\{\varphi\}$, i.e. after $\operatorname{claim}(\varphi)$ the formula $\varphi$ is added to the hypothetical commitment base, [E2] if $s\left(m_{n}\right)=w h y(\varphi)$, then $C_{s}^{\prime}\left(m_{0}, \ldots, m_{n}\right)=C_{s}^{\prime}\left(m_{0}, \ldots, m_{n-1}\right)$, [E3] if $s\left(m_{n}\right)=$ concede $(\varphi)$, then $C_{s}^{\prime}\left(m_{0}, \ldots, m_{n}\right)=C_{s}^{\prime}\left(m_{0}, \ldots, m_{n-1}\right) \cup\{\varphi\},[\mathbf{E} 4]$ if $s\left(m_{n}\right)=(\varphi \vee \psi)$ since $\varphi$, then $C_{s}^{\prime}\left(m_{0}, \ldots, m_{n}\right)=C_{s}^{\prime}\left(m_{0}, \ldots, m_{n-1}\right) \cup\{\varphi\}$, i.e. after $(\varphi \vee \psi)$ since $\varphi$ the formula $\varphi$ is added to $s$ 's hypothetical commitment base, [E5] if $s\left(m_{n}\right)=$ question $(\varphi)$, then $C_{s}^{\prime}\left(m_{0}, \ldots, m_{n}\right)=C_{s}^{\prime}\left(m_{0}, \ldots, m_{n-1}\right)$. 